\title{
Vectores de la leishmaniasis tegumentaria en el valle del Rímac
}

\author{
ABELARDO TEJADA, ABRAHAM CÁCERES, JUAN MIRANDA, OLGA PALACIOS, VÍCTOR ZORRILLA \\ Instituto de Medicina Tropical "Daniel A. Carrión”-UNMSM.
}

\begin{abstract}
RESUMEN
OBJETIVO: Determinar la composición de especies de Lutzomyia en el valle del Rímac. MATERIAL Y MÉTODOS: El área estudiada fue el anexo de Ullupampa (2000 msnm), ubicado a 1 kilómetro de Surco, distrito de San Jerónimo de Surco, provincia de Huarochirí, Lima. Para la captura de flebotominos se utilizó trampa Shannon, trampas de luz CDC y captura directa con aspirador manual, en ambientes intra y peridomiciliarios y refugios naturales extradomiciliarios. RESULTADOS: Se identificó 1742 flebotominos del género Lutzomyia, de las cuales $52,6 \%$ corresponden a Lutzomyia verrucarum, 28,2\% a Lutzomyia peruensis y $19,2 \%$ a Lutzomyia noguchi, las primeras abundantes en intra y peridomicilio y la última en medios extradomiciliarios. La densidad de Lutzomyia presenta variaciones estacionales, siendo mayor entre los meses de marzo y mayo. La actividad hematófaga horaria presenta un pico entre las 20 y 21 horas. CONCLUSIONES: Las viviendas rodeadas de cultivos de plantas frutales separadas por "pircas" y animales domésticos en el intradomicilio, favorecen la actividad hematófaga domiciliaria de L. peruensis y L. verrucarum, principales vectores de la leishmaniasis en el valle del Rímac.
\end{abstract}

Palabras clave: Leishmaniasis; psychodidae; phlebotomus.

\section{CUTANEOUS LEISHMANIASIS VECTORSSTUDY AT RIMACVALLEY SUMMARY}

OBJECTIVES: To determine the composition of Lutzomyia species at the Rimac valley. MATERIAL AND METHODS: The area studied was the Ullupampa annex (2000 msnm), San Jeronimo de Surco district, Huarochiri province, Lima. The sandflies were captured using Shannon traps, CDC light traps and direct capture, in intra and peridomiciliary ambient and natural refuges. RESULTS: We identified 1742 phlebotomine sandflies of the Lutzomyia genus, $52,6 \%$ corresponded to Lutzomyia verrucarum, $28,2 \%$ to Lutzomyia peruensis, and $19,2 \%$ to Lutzomyia noguchi; the first two were more abundant in intra and peridomiciliary ambients, and the latest in natural refuges. Lutzomyia density presents seasonal variations, mostly found between March and May. Night activity peak is between 20 and 21 hours. CONCLUSIONS: Fruit cultivations surrounded housings separated by "pircas" and domestic animals within the house favor $L$. peruensis and L. verrucarum hematophagous activity in the domiciliary ambient; they represent the main leishmaniasis vectors at Rimac valley.

Key words: Leishmaniasis; psychodidae; phlebotomus.

\footnotetext{
Correspondencia:

Dr. Abelardo Tejada Valencia

Instituto de Medicina Tropical "Daniel A. Carrión"

UNMSM - Sección de Parasitología.

Jr. José Santos Chocano 199. Urb. San Joaquín

Callao 02, Perú

E-mail: abelardo_tejada@hotmail.com
} 


\section{INTRODUCCIÓN}

En el Perú, hasta el momento se ha descrito 131 especies de Lutzomyia, de las cuales cinco son vectores de leishmaniasis tegumentaria $\left({ }^{1,2}\right)$. Lutzomyia peruensis (Shannon, 1929) es el vector natural de la "uta" en las provincias de Huarochirí, Otuzco y Bolognesi ( $\left.{ }^{3-6}\right)$. Lutzomyia ayacuchensis (Cáceres \& Galati, 1988) es el vector de la leishmaniasis cutánea andina en las provincias de Lucanas y Parinacochas $\left(^{7}\right)$. Lutzomyia verrucarum (Townsend, 1913) es probable vector de leishmaniasis en algunas áreas endémicas de las provincias de Bolognesi y Huarochirí ( $\left.{ }^{5,6}\right)$. Lutzomyia tejadai (Galati \& Cáceres, 1990) ha sido encontrada infectada naturalmente con Leishmania spp. en las provincias de Ambo y Huánuco. Lutzomyia pescei se correlaciona geográficamente con algunas áreas endémicas de leishmaniasis ( ${ }^{1}$ ).

En el valle del Rímac, la leishmaniasis coexiste con la bartonelosis humana y se distribuye desde los 1000 hasta los $3000 \mathrm{msnm}$. En esta zona se ha identificado 3 especies de Lutzomyia, de las cuales Lutzomyia peruensis y L. verrucarumson altamente antropofílicas, mientras que L. noguchi sólo pica a pequeños roedores $\left({ }^{3}\right)$. En los últimos años, se han presentado numerosos casos de leishmaniasis en el valle $\left({ }^{8}\right)$.

El objetivo del presente estudio fue determinar los factores ecológicos que contribuyen a la presencia de Lutzomyia en esta zona, la densidad estacional, así como la actividad horaria.

\section{MATERIAL Y MÉTODOS}

El estudio se realizó en la localidad de Ullupampa, área rural ubicada a $2000 \mathrm{msnm}$ en la margen izquierda del río Rímac, a un kilómetro del centro urbano de Surco, distrito de San Jerónimo de Surco, provincia de Huarochirí, Lima. Las viviendas están construidas de adobe con techo de calamina y los terrenos de cultivo están separados por "pircas". La actividad principal de los pobladores de la zona es la agricultura (árboles frutales, hortalizas) y la crianza de animales domésticos (cabras, cobayos, aves de corral y perros).

De enero de 1996 a diciembre de 1997, se realizó capturas de flebotominos, cinco días cada mes, en ambientes intradomiciliarios (dormitorios, cuyeros), peridomiciliarios (corrales de animales domésticos) y en campo abierto (cuevas de roedores), utilizando trampas de luz tipo CDC (desde las 18:00 hasta las 06:00 h), trampa tipo Shannon (desde las 19:00 hasta las 01:00 h) y captura directa con aspirador manual, linterna de mano y humo de cigarrillo $\left({ }^{9-11}\right)$.

La identificación de los flebotominos se realizó utilizando claves dicotómicas y descripciones originales $\left({ }^{12-14}\right)$.

\section{RESULTADOS}

Se capturó 1742 flebotominos del género Lutzomyia (30,7\% machos y 69,4\% hembras), pertenecientes a tres especies, siendo $L$.verrucarum $(52,6 \%)$ la más abundante, L. peruensis representa $28,2 \%$ yL. noguchi $19,2 \%$ (Tabla 1 ).

En la Tabla 2 se puede observar que las especies L. verrucarum y L. peruensis son las predominantes en las capturas con trampas de luz CDC y trampa Shannon, mientras que

Tabla 1.- Especies de Lutzomyia spp. capturados.

\begin{tabular}{lrrrr}
\hline Especies & Machos & Hembras & \multicolumn{2}{c}{ Total } \\
\cline { 3 - 5 } & & & $\mathrm{n}$ & $\%$ \\
\hline L. verrucarum & 158 & 758 & 916 & 52,6 \\
L. peruensis & 88 & 403 & 491 & 28,2 \\
L. noguchi & 288 & 47 & 335 & 19,2 \\
Total & 534 & 1208 & 1742 & \\
$\%$ & 30,7 & 69,4 & & \\
\hline
\end{tabular}


Tabla 2.- Densidad se Lutzomyia sp. por metodo de captura.

\begin{tabular}{|c|c|c|c|c|c|c|c|c|}
\hline \multirow[b]{2}{*}{ Especies } & \multicolumn{2}{|c|}{ Shannon } & \multicolumn{2}{|c|}{$\mathrm{CDC}$} & \multicolumn{2}{|c|}{ Directo } & \multicolumn{2}{|c|}{ Total } \\
\hline & $\mathrm{M}$ & $\mathrm{H}$ & $\mathrm{M}$ & $\mathrm{H}$ & $\mathrm{M}$ & $\mathrm{H}$ & $\mathrm{M}$ & $\mathrm{H}$ \\
\hline L. verrucarum & 38 & 375 & 67 & 316 & 53 & 67 & 158 & 758 \\
\hline L. peruensis & 33 & 203 & 34 & 119 & 21 & 81 & 88 & 403 \\
\hline L. noguchi & 1 & 0 & 0 & 2 & 287 & 45 & 288 & 47 \\
\hline Total & 72 & 598 & 101 & 417 & 361 & 193 & 534 & 1208 \\
\hline
\end{tabular}

M: Machos

H: Hembras

L. noguchi es más abundante en las capturas directas. $L$. verrucarum es más abundante en medios intradomiciliarios seguida de L. peruensis, mientras que $L$. noguchi presenta alta densidad en ambientes naturales extradomiciliarios (Figura 1). La actividad antropofílica horaria presenta un pico entre las 20 y 21 horas y disminuye a medida que desciende la temperatura y se incrementa la humedad relativa (Tabla 3, Figura 2), mientras que la más alta densidad poblacional se presenta de marzo a mayo (Figura 3 ).

\section{DISCUSIÓN}

Lutzomyia peruensis es el vector natural de la leishmaniasis en las provincias de Huarochirí, Otuzco y Bolognesi ( $\left.{ }^{3-6}\right)$. En la localidad de Surco, esta especie coexiste con Lutzomyia verrucarum, vector natural de la bartonelosis humana $\left({ }^{10}\right)$. Estas dos especies son altamente antropofílicas y zoofílicas, lo que sugiere que ambas estarían participando en la transmisión de la leishmaniasis tegumentaria en esta zona. Lutzomyia verrucarumes un vector competente para transmitir la leishmaniasis al hombre, ya que ha sido encontrada infectada naturalmente con Leishmania del complejobraziliensisen las provincias de Huarochirí y Bolognesi $\left({ }^{6,15}\right)$.

La densidad poblacional de estas dos especies presenta variaciones estacionales, así como su actividad horaria, lo que sugiere que la tasa más alta de transmisión ocurre durante
Tabla 3.- Promedios horarios de temperatura y humedad relativa y su influencia en la actividad hematofaga de Lutzomyia spp.

\begin{tabular}{cccc}
\hline Hora & Temperatura & HR $(\%)$ & Lutzomyia spp. $(\%)$ \\
\hline $19-20$ & 17,5 & 70 & 8,9 \\
$20-21$ & 16,5 & 74 & 37,8 \\
$21-22$ & 15,0 & 80 & 16,3 \\
$22-23$ & 14,0 & 83 & 15,1 \\
$23-00$ & 13,5 & 88 & 13,6 \\
$00-01$ & 13,0 & 90 & 8,4 \\
\hline
\end{tabular}

HR: Humedad relativa.

los meses de marzo, abril y mayo, y la probabilidad de adquirir la enfermedad es mayor durante las primeras horas de la noche, mientras las personas duermen o descansan en el peridomicilio. Lutzomyia noguchi no pica al hombre y sólo se alimenta de pequeños roedores, pero probablemente cumpla un rol importante en el mantenimiento de la leishmaniasis en la naturaleza $\left({ }^{16}\right)$. La distribución altitudinal de los flebotominos capturados en el valle, está dentro de los límites donde la leishmaniasis es endémica $\left({ }^{17}\right)$.

Entre los factores ecológicos que determinan la presencia del género Lutzomyia en el valle del Rímac, tenemos: a) clima favorable durante todo el año, con temperatura media anual de $20^{\circ} \mathrm{C}$ y una humedad relativa variable entre 60 y $95 \%, b)$ presencia de animales domésticos y 


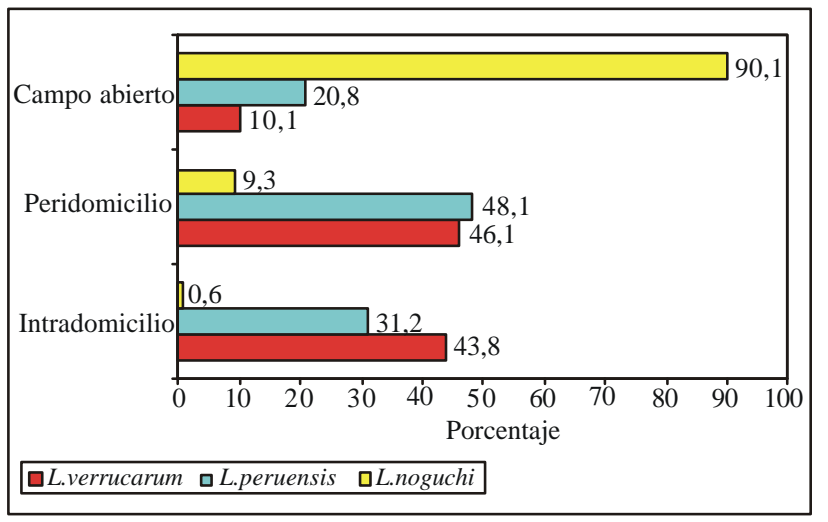

Figura 1.- Capturas en ambientes intra, peri y extradomiciliarios.

silvestres en el medio domiciliario, probables reservorios del parásito, c) la vegetación que rodea a las viviendas y las "pircas", constituyen un refugio natural para los flebotominos.

\section{BIBLIOGRAFÍA}

1. Cáceres A. Especies de Lutzomyia (Diptera: Psychodidae, Phlebotominae) vectores de la "uta" en el Perú. Rev Per Ent 1995; 38: 23-6.

2. Cáceres A, Galati E, Pinto J, Paredes R, Reátegui R, Pérez J, et al. Psychodidae (Diptera) del Perú I: Phlebotominae en Huánuco, Pasco y Cusco, su relación con

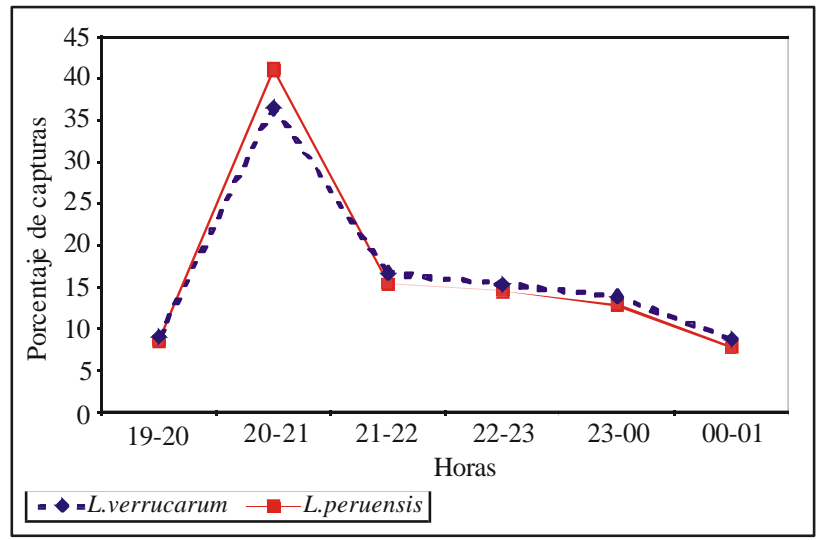

Figura 2.- Actividad hematofaga horaria de $L$. peruensis y L. verrucarum. la enfermedad de Carrión y la leishmaniosis tegumentaria. Rev per Biol 2000; 7(1): 27-43.

3. Herrer A. Lutzomyia peruensis (Shannon, 1929) posible vector natural de la "uta" (leishmaniasis tegumentaria). Rev Inst Med Trop Sao Paulo 1982; 24: 178-82.

4. Cruzado L. Lutzomyia peruensis vector de Leishmania spp. en Pagash, Otuzco, Perú. Resúmenes de Trabajos Científicos, X Congreso Latinoamericano de Microbiología. Trujillo 1987; 9: 187.

5. Pérez J, Villaseca P, Cáceres A, López M, Zolessi A, Campos M, et al. Leishmania (Viannia) peruviana isolated from the sandfly Lutzomyia peruensis (Diptera: Psychodidae) and a sentinel hamster in the Huayllacallan Valley, Ancash, Peru. Trans Roy Soc Trop Med Hyg 1991; 85: 60.

6. Pérez E, Ogusuku E, Inga R, López M, Monje J, Paz L, et al. Natural Leishmania infection of Lutzomyia spp. in Peru. Trans Roy Soc Trop Med Hyg 1994; 88: 161-4.

7. Cáceres A, Villaseca $\mathbf{P}$, Inga $\mathbf{R}$, López $\mathbf{M}$, Arévalo J, Llanos-Cuentas A. Lutzomyia ayacuchensis (Diptera: Psychodidae, Phlebotominae) probable vector de la leishmaniasis cutánea andina (uta) en Lucanas y Parinacochas (Ayacucho, Perú). V Congreso Panamericano Infectología, Lima 1991; p. 22.

8. Méndez R, Medina V, Moscoso M. Epidemiología de la leishmaniasis en la Sub-Región de Salud IV, Lima Este. V congreso peruano de enfermedades infecciosas y tropicales 1997; 6(2): 69.

9. Pérez E, Villaseca P, Llanos-Cuentas A, Campos M, Guerra H. Técnicas para colectar "titiras" (Lutzomyia spp., Diptera: Psychodidae) en ambientes altoandinos. Rev Per Ent 1987; 30: 77-80.

10. Cáceres A. Distribución geográfica de Lutzomyia verrucarum (Towsend, 1913) (Diptera, Psychodidadae, Phlebotominae) vector de la bartonelosis humana en el Perú. Rev Inst Med Trop Sao Paulo 1993; 35(6): 485-90.

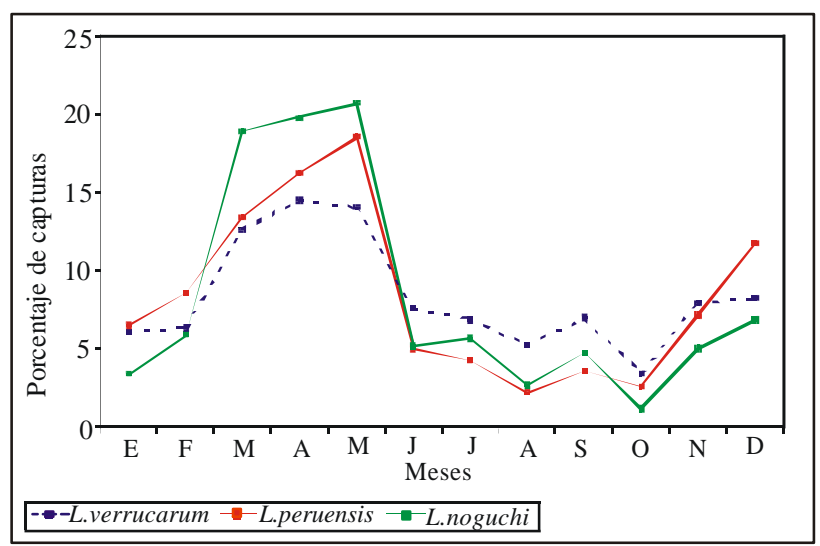

Figura 3.- Actividad estacional de Lutzomyia spp. 
11. Tejada A. Leishmaniasis tegumentaria en el Perú. Observaciones entomológicas en Manu, Madre de Dios. Rev Per Epidem 1993; 6(2): 40-2.

12. Forattini O. Entomología Médica. IV: Psychodidae, Phlebotominae, Leishmanioses, Bartonelose. Sao Paulo 1973.

13. Galati EAB. Sistemática dos phlebotominae (Diptera, Psychodidae) das Américas. Tese de Doutoramento apresentada ao Departamento de Epidemiología da Facultade de Saúde Pública da USP. Sao Paulo 1990; p. 275.

14. Young D, Duncan M. Guide to the identification and geographic distribution of Lutzomyia sand flies in Mexico, the West Indies, Central and South America (Diptera: Psychodidae). Mem of the Amer Ent Inst 1994; 54: 1-881.
15. Davies C, Fernández M, Paz L, Roncal N, LlanosCuentas A. Lutzomyia verrucarum can transmitLeishmania peruviana, the aetiological agent of Andean cutaneous leishmaniasis. Trans Roy Soc Trop Med Hyg 1993; 87: 603-6.

16. Davies C, Llanos-Cuentas A, Campos P, Monje J, Villaseca P, Dye C. Cutaneous leishmaniasis in the peruvian Andes: risk factors identified from a village cohort study. Am J Trop Med Hyg 1997; 56(1): 85-95.

17. Villaseca P, Llanos-Cuentas A, Pérez E, Davies C. A comparative field study of the relative importance of Lutzomyia peruensis and Lutzomyia verrucarum as vectors of cutaneus leishmaniasis in the peruvian Andes. Am J Trop Med Hyg 1993; 49(2): 260-9. 